the outside back cover but also in the extensive revision of all sections of the book. An emphasis on holistic "assessment" rather than "examination", the role of the doctor in the multi-agency team and the advice to seek specific consent for ano-genital examination all help to bring the book up to date.

New chapters on bruises, ophthalmic presentations and visceral injuries as well as the chapters on burns and scalds, fractures and head injuries benefit from reference to the evidence base and further reading. There are useful check lists in the chapters on neglect, non-organic failure to thrive and emotional abuse. Throughout the book there are reminders to consider differential diagnosis, including conditions that mimic bruising (a photograph of a Mongolian blue spot would have been helpful here) and the misdiagnosis of neglect.

The book is well illustrated by case histories and pictures, which are of generally high quality apart from some rather disappointing reproductions in the chapter on bruising. There is some repetition, mainly of essential information, for the benefit of readers consulting individual chapters. However, the three chapters on sexual abuse would have benefited from more careful editing to avoid an out of place section on examination technique at the end of the chapter on interpretation of findings and a second rather dated picture of a colposcope.

Although the $\mathrm{ABC}$ series is written primarily for general practitioners, child abuse can present in many different ways to any specialist. This book will succeed in its aim to raise awareness in all doctors and increase recognition of abuse as well as being useful for non-medical professionals to understand the strengths and weaknesses of medical reports. It is a concise, well balanced and easy to use practical guidebook containing a wealth of accessible information.

In the current litiginous climate, many paediatricians are, not surprisingly, reluctant to become involved in child protection but we all need to know how to manage these situations if only to avoid the possibility of a clinical negligence claim by the child in the future if we fail to take appropriate action. Roy Meadow may no longer be allowed to give his opinion in child-protection cases but through this book we can access his experience and knowledge. For me, his final chapter on Dilemmas was both reassuring and encouraging and should give confidence both to the inexperienced and to those reluctant to open the can of worms.

Child-protection training is mandatory; this book should be compulsory reading for all doctors who come into contact with children.
Angela Moore

Wolverhampton City PCT, The Gem Centre, Neachells Lane Wolverhampton, WV11 3PG; angela.moore@wolvespct. nhs.co.uk

\section{Practical endocrinology and diabetes in children, 2nd edition}

Edited by Joseph E Raine, Malcolm D C Donaldson, John W Gregory, Martin 0 Savage and Raymond L Hintz. Published by Blackwell, Oxford, 2006, pp 256, £59.99 (hardback). ISBN 1405122331

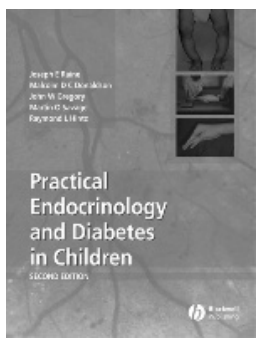

I once won a prize in a "scientific writing" competition. We did not know the criteria by which we were going to be judged, and I was very surprised to hear that I had won. Soon all was revealed: the prize was for the shortest sen-

tence. Our tutor felt that concise writing was important in science. I was reminded of it reading this book. Although some topics included are mightily complicated, the sentences tend not to be. Despite there being five authors there is a remarkable uniformity in style. The whole book is written in clear and concise way, and at the same time it is comprehensive and covers all the necessary topics.

In the preface, the authors state that the aim of the book is to "bridge the gap between the large detailed endocrine reference book and the short review of aspects of endocrinology". The book is what the title says: a practical guide. The chapters are problem-based and give an overview of the relevant physiology, followed by a practical guide to examination, investigation and management. Within the structure of each chapter, there are headings such as "controversial points", "when to involve a specialist centre", "future developments", "case histories" and "potential pitfalls". All this makes it a very didactic book where for a given problem you are led through the background information on the topic, things you need to look out for in history, examination and management. There are a number of management protocols including an up to date diabetic ketoacidosis (DKA) protocol and "guidelines for follow-up".

As a paediatrician in a general hospital, I am exactly the person this book is primarily aimed at. I have tested it on a number of issues, most recently during a weekend on call when managing a child with a very complex history and suspected adrenal insufficiency. The book had a list of what bloods to take, a table with the doses of hydrocortisone replacement and a copy of a CAH therapy card designed for a congenital adrenal hyperplasia (CAH) patient that, after some adaptation, I found a useful starting point for the information I needed to give to the parents. Just to be sure, I reached for my copy of Sperling's Pediatric endocrinology to look up the same subject. I have always liked that book and have learned a lot during the times I have spent with it but the contrast was stark. What (and why) I needed to do was spelled out clearly in Practical Endocrinology and Diabetes in Children, while Sperling's book was overwhelming and I struggled to find quickly the practical clinical pearls of information I needed. For the busy times and practical answers to clinical scenarios this book is unbeatable.

The layout of the text with many bullet points, tables and diagrams makes the book extremely readable. The book is printed on slightly glossy paper, which aids clarity and is useful as the font is smaller than average. Introduction of colour within the text and diagrams would be great. Interestingly, the management guidance points are not referenced individually. Rather, the authors present established current practice, and at the end of each chapter there are general references and key papers. There is an appendix with a list of UK and North American patient support groups, various growth charts (no height velocity chart) and a CAH therapy card.

I have a very high opinion of this book. It is one of the best clinical books I have ever come across.

Ewa Posner University Hospital of North Durham, UK; e.b.posner@ncl.ac.uk

\section{CORRECTION}

doi:10.1136/adc.2007.136051corr1

Fry T. If it's worth doing, let's do it! Arch Dis Child 2008;93:267-8. The following competing interest statement should have been included with this article.

Under an agreement with the UK Charities Commission, Novo Nordisk UK (who make growth hormone therapies) fund a proportion of Mr Fry's salary so that he can run the Child Growth Foundation (CGF) full time - he receives no direct financial support from the CGF. The foundation receives ah hoc fees from a number of drugs companies who buy the auxology training course. In addition CGF manages certain research projects for clinicians that are funded by drug companies (CGF receives no management fee but profits from the bank interest accrued while the research is being conducted). 